\title{
ACTAS DE LAS JORNADAS SOBRE RIESGOS INHERENTES A LA DIGITALIZACIÓN DEL TRABAJO: NUEVOS HORIZONTES PARA LA PREVENCIÓN DE RIESGOS LABORALES
}

\author{
JAVIER FERNÁNDEZ-COSTALES MUÑIZ \\ Catedrático EU (integrado TU) de Derecho del Trabajo y de la Seguridad Social \\ ROBERTO FERNÁNDEZ FERNÁNDEZ \\ Profesor Titular de Derecho del Trabajo y de la Seguridad Social
}

Universidad de León

En la Facultad de Derecho de la Universidad de León se celebraron los días 12, 13, 17 y 18 de septiembre de 2018 las "Jornadas sobre riesgos inherentes a la digitalización del trabajo: nuevos horizontes para la Prevención de Riesgos Laborales", y que a lo largo de cuatro jornadas, bajo la dirección de los Dres. Javier Fernández-Costales Muñiz y Roberto Fernández Fernández, reunieron a profesores de la Universidad de León con expertos de otras universidades que disertaron en torno a la digitalización en el ámbito laboral y su influencia en materia de Prevención de Riesgos Laborales.

La primera jornada se abrió con la ponencia de uno de los Directores de las Jornadas, el Prof. Roberto Fernández Fernández en torno a "Las adicciones provocadas por la utilización de las nuevas tecnologías: ¿nuevos factores de riesgo psicosocial?”, a quien siguió la intervención de la Prof a . Susana Rodríguez Escanciano, quien abordó una cuestión da tanta actualidad e importancia como "El tratamiento de datos sensibles vinculados a la prevención de riesgos laborales". Las exposiciones se completaron desde un punto de vista del Derecho Administrativo en este ámbito, con la participación de los Profs. Tomás Quintana López y Ana Belén Casares Marcos, quienes disertaron sobre los "Mecanismos jurídicos de prevención ambiental en actividades de la industria extractiva" y "El tratamiento de los datos preventivos en el marco de la Administración electrónica”, respectivamente.

La segunda jornada incluyó la impartición de las conferencias del Prof. Juan José Fernández Domínguez sobre "Prevención de Riesgos Laborales causados por las nuevas tecnologías", el Prof. José Gustavo Rodríguez Quirós habló de los "Riesgos asociados a la utilización de pantallas de visualización y dispositivos de control", mientras el Prof. Rodrigo Tascón López disertó sobre los “Accidentes en misión e in itinere en el marco de la industria 4.0", finalizando el día con la presencia de la Catedrática de Derecho del Trabajo y de la Seguridad Social de la Universidad 
Complutense Yolanda Sánchez-Urán Azaña quien cerró las ponencias con el tema "Implicaciones de la robótica en la seguridad en el trabajo".

El tercer día del Seminario se presentaron cuatro nuevas exposiciones temáticas sobre la materia, comenzando la Prof ${ }^{\mathrm{a}}$. Henar Álvarez Cuesta, quien desarrolló los aspectos relativos a los "Trabajadores con discapacidad e industria 4.0: ¿nuevos riesgos laborales o una oportunidad para la inclusión", seguida por el Prof. Javier FernándezCostales Muñiz, que abordó la cuestión de "La prevención de los nuevos riesgos laborales en los trabajadores migrantes". La segunda parte de la jornada fue abordada por parte del equipo decanal de la Facultad de Ciencias del Trabajo de la Universidad de León, al tratar las Prof ${ }^{\mathrm{a}}$. Beatriz Agra Viforcos y María de los Reyes Martínez Barroso las cuestiones en torno al "Género y prevención de riesgos en la industria 4.0" y "La prevención de los riesgos laborales de los trabajadores autónomos y las nuevas tecnologías", respectivamente.

Para el cierre de las Jornadas se desarrolló una mesa redonda sobre la "Digitalización y prevención de riesgos laborales en Castilla y León”, integrada por los Profes. Javier Fernández-Costales Muñiz, Roberto Fernández Fernández, Natalia Ordóñez Pascua y Francisco Xabiere Gómez García, que dió paso a la conferencia de clausura, pronunciada por el Prof. Ignacio Gonzáles del Rey Rodríguez, Catedrático de Derecho del Trabajo de la Universidad de Oviedo, quien expuso "Los procesos sobre responsabilidad por incumplimientos en materia de Prevención de Riesgos Laborales", finalizando con las conclusiones expuestas por los Directores del evento.

El amplio e interesante contenido de las conferencias pronunciadas en el marco de las Jornadas se resume a continuación con las aportaciones realizadas por los propios ponentes.

Ponencia: LAS ADICCIONES PROVOCADAS POR LA UTILIZACIÓN DE LAS NUEVAS TECNOLOGÍAS: ¿NUEVOS FACTORES DE RIESGO PSICOSOCIAL? Ponente: Roberto Fernández Fernández, Profesor Titular de Derecho del Trabajo y de la Seguridad Social de la Universidad de León

Las opciones vinculadas a la utilización de nuevas herramientas tecnológicas constituye un avance significativo en las sociedades actuales y permiten unas posibilidades de intercambio hasta ahora desconocidas. Las posibilidades de comunicación se han incrementado de manera exponencial como consecuencia de tener al alcance de manera inmediata tanto dispositivos como instrumentos que permiten interactuar con otras personas de manera instantánea y sencilla.

Por otra parte, la generalización de redes sociales como twitter, whatssap, facebook, snapchat, linkedin, etcétera, ha traído consigo importantes beneficios pero también retos desde el punto de vista jurídico, tendentes a ordenar las posibles conductas que pueden ser realizadas a través de las mismas. 
Dichos comportamientos acaban por incidir tanto en la faceta personal como laboral de las personas, pues la utilización continua, habitual y reiterada de las nuevas tecnologías está cambiando la forma de actuar de los seres humanos. Tal circunstancia también presenta sus consecuencias a nivel laboral, obligando a realizar ciertas adaptaciones en el marco de la organización productiva para intentar adaptar esta a la nueva realidad y, como no, a abrir cierto debate sobre si es necesaria o no cierta intervención jurídica para intentar poner remedio a algunas de los problemas vinculadas al uso de las nuevas tecnologías en el lugar de trabajo.

En este sentido, ya se están comenzado a estudiar determinados comportamientos vinculados al uso de estas redes sociales y que pueden traer aparejadas alteraciones de la salud de quienes las utilizan, dando lugar a términos como tecnofobia (u odio a las nuevas tecnologías), tecnoansiedad (o negatividad ante las mismas por incapacidad para su manejo), tecnoadicción (o necesidad imperiosa de un uso continuado de estas herramientas) y tecnofatiga (hartazgo por la utilización permanente de las mismas).

Las condiciones laborales pueden coadyuvar, junto con otros factores, a magnificar los perjuicios de las consecuencias anteriormente expuestas, en tanto en cuanto la organización productiva puede querer del trabajador estar permanentemente conectado a través de las nuevas tecnologías a su actividad productiva (no se olvide que las herramientas tecnológicas facilitan enormemente el estar disponible y localizable casi las 24 horas del día) intentando con ello dar al cliente el mejor de los servicios y ganar su confianza aumentando con ello la fidelidad del mismo y mejorar su cuenta de resultados. Sin duda alguna dicha situación va a constituir un factor de estrés importante que puede causar trastornos físicos y psíquicos en el trabajador, habida cuenta de que va a provocar llamadas o conexiones a horas fuera del horario laboral, sobrecarga de trabajo, adicción al mecanismo tecnológico al tener que estar pendiente constantemente de él o controles no autorizados por parte del empleador.

Para paliar dicha situación, y evitar que el trabajador tenga la obligación de estar conectado permanentemente a través de las nuevas tecnologías con su quehacer productivo, se ha introducido en el foro jurídico el debate sobre la garantía del denominado "derecho a la desconexión" que algunos países, como Francia, ya han incorporado a su ordenamiento jurídico y que pretende garantizar el descanso correspondiente del trabajador, forzando a las empresas a poner en marcha políticas de modernización tecnológica que garanticen los derechos de los trabajadores.

Ponencia: EL TRATAMIENTO DE DATOS SENSIBLES VINCULADOS A LA PREVENCIÓN DE RIESGOS LABORALES

Ponente: Susana Rodríguez Escanciano, Catedrática de Universidad de Derecho del Trabajo y de la Seguridad Social de la Universidad de León

La progresiva y vertiginosa irrupción de la tecnología digital, telemática, robótica, plataformas, algoritmos, internet de las cosas, comunicaciones máquina a máquina, realidad aumentada e inteligencia artificial en la actividad productiva bajo la denominación de industria 4.0, permite no sólo optimizar y agilizar el desempeño de 
la prestación profesional sino también almacenar una cantidad enorme de información relativa a la persona del trabajador que se entremezcla a su vez con la prestación laboral. Sin duda, la gestión informatizada del personal facilita que todos los datos concernientes al desarrollo del contrato de trabajo, desde el momento de la selección de personal, pasando por la constitución del vínculo contractual hasta su resolución, sean incluidos en los soportes informáticos de la empresa; sin olvidar tampoco cómo la incorporación de la persona del trabajador a la organización productiva empresarial favorece tanto una continua adquisición de información sobre extremos personales de diversa naturaleza como su minuciosa puesta al día.

Desde tales premisas, cobra especial relevancia la necesidad de proteger el interés legítimo del trabajador de controlar sus datos personales insertos en los sistemas de comunicación empresariales porque sólo así va a poder evitar una afectación negativa durante la relación laboral, máxime si se trata de datos especialmente sensibles, obtenidos en el cumplimiento de las obligaciones relacionadas con la prevención de riesgos laborales principalmente a través de la realización de los pertinentes reconocimientos médicos.

Como fácilmente puede intuirse, los reconocimientos médicos permiten, mediante la realización de las indagaciones, las pruebas clínicas y las averiguaciones biológicas o biométricas, la evaluación del estado de salud del trabajador, el conocimiento de sus posibles patologías y su origen y, también, la aplicación de los oportunos remedios en orden a un eventual restablecimiento del bienestar. Desde la óptica del cumplimiento de la obligación empresarial de proporcionar seguridad en el trabajo, los resultados de los reconocimientos médicos permitirán planificar $\mathrm{y}$, en su caso, reorientar la actividad preventiva en la empresa. Ahora bien, ocurre que el reconocimiento médico implica, en sí mismo, una intromisión en la esfera privativa del trabajador. Tal contraposición de intereses exige la búsqueda de un punto de equilibrio.

A la espera de la respuesta expresa del legislador español, tras un fracasado Proyecto de Ley, es necesario analizar los principios y garantías recogidos en el Reglamento UE 2016/679 del Parlamento europeo y del Consejo de 27 de abril de 2016, de Protección de Datos Personales, sin olvidar tampoco las pautas introducidas al respecto en la Ley 31/1995, de 8 de noviembre, de Prevención de Riesgos Laborales, que acoge como postulado básico la autonomía de la persona del trabajador para someterse o no a los reconocimientos médicos, propuestos por la empresa, permitiendo, en su caso, exploraciones y analíticas sobre datos corporales, o impidiendo pruebas clínicas ajenas a la finalidad de la vigilancia de la salud en relación con los riegos inherentes al trabajo. Este criterio se conecta directamente con la obligación de información previa, de forma que el trabajador debe ser expresamente alertado sobre los exámenes médicos especialmente invasores de su intimidad al tiempo de otorgar su consentimiento. 
Ponencia: MECANISMOS JURÍDICOS DE PREVENCIÓN AMBIENTAL EN ACTIVIDADES DE LA INDUSTRIA EXTRACTIVA

Ponente: Tomás Quintana López, Catedrático de Derecho Administrativo de la Universidad de León

El tema a tratar desde el punto de vista jurídico es complejo debido a que las soluciones que ofrece el ordenamiento a la tensión entre la minería y el medio ambiente vienen establecidas en diversas normas jurídicas, unas concebidas para ser aplicadas de forma transversal, es decir a diferentes actividades, entre las que se hallan también las actividades mineras; y otras específicamente concebidas para ser aplicadas al sector minero.

La exposición consta de dos partes: una primera relativa a los antecedentes legislativos y su aplicación, y una segunda dedicada al régimen jurídico actual, integrado, como se ha indicado, por normas de proyección trasversal y por otras específicamente previstas para las actividades mineras.

I. Antecedentes normativos y su aplicación

1. Previsiones de la Ley de Minas (1973)

2. Reglamento de Actividades Molestas, Insalubres, Nocivas y Peligrosas, aprobado por el Decreto 2414/1961, de 30 de diciembre, y su contradictoria aplicación con los artículos 116 y 117 de la Ley de Minas. Solución jurisprudencial

3. La Constitución y su influencia en la regulación de la tensión entre la minería y el medio ambiente:

A) Ley de Cataluña 12/1981, de 24 de diciembre, por la que se establecen normas adicionales de protección de los espacios de especial interés natural afectados por actividades extractivas// STC 64/1982, de 4 de noviembre.

B) La restauración de los espacios afectados por actividades extractivas. Reales Decretos 2.994/1982, de 15 de octubre, y 1.116/1984, de 9 de mayo.

II. Régimen actual

1. Legislación transversal. Controles administrativos previos al desarrollo de las actividades mineras por razones ambientales: Evaluación de Impacto ambiental y prevención y control integrado de la contaminación (autorización ambiental integrada). Coordinación de ambos controles.

2. Restauración de espacios afectados por actividades extractivas y gestión de residuos.

A) Real Decreto 975/2009, de 12 de junio, sobre gestión de los residuos de las actividades extractivas y de protección y rehabilitación del espacio afectado por actividades extractivas.

B) El Plan de Restauración. Contenido, aspectos procedimentales, ejecución del plan de restauración y régimen sancionador. 
Ponencia: EL TRATAMIENTO DE LOS DATOS PREVENTIVOS EN EL MARCO DE LA ADMINISTRACIÓN ELECTRÓNICA

Ponente: Ana Belén Casares Marco, Catedrática Acreditada de Derecho Administrativo de la Universidad de León

La aprobación y entrada en vigor de las Leyes 39/2015, de 1 de octubre, del Procedimiento Administrativo Común de las Administraciones Públicas, y 40/2015, de 1 de octubre, de Régimen Jurídico del Sector Público, ha desatado una honda preocupación acerca de sus implicaciones concretas tanto para los operadores jurídicos, públicos y privados, como para los ciudadanos en general. Inician una nueva etapa en relación con la organización y el funcionamiento del sector público español, marcada, fundamentalmente, por la separación en dos textos normativos diversos de la regulación de las vertientes ad extra y ad intra de nuestras Administraciones públicas, destacando, en particular, la preocupación del legislador, cuando no su auténtica obsesión, por continuar profundizando en la Administración electrónica. La reforma incorpora, en tal sentido, importantes innovaciones, escudándose en la necesidad de mejorar el funcionamiento interno de nuestra Administración pública y, en particular, en los principios de eficacia y eficiencia, para implantar un nuevo modelo administrativo que suscita, sin duda, cuestiones y objeciones que precisan respuesta.

En efecto, no cabe desconocer que la fiebre informática que caracteriza a nuestra sociedad contemporánea conlleva un grave riesgo para algunos de los derechos fundamentales más importantes que reconoce nuestra Constitución. La evolución constante e imparable de las nuevas tecnologías, su prácticamente ilimitado potencial para el tratamiento de la información y, en particular, de los datos personales, supone, en este sentido, una amenaza cierta que pone en serios aprietos la capacidad de los ciudadanos de controlar toda aquella información personal que se refiera a ellos o les afecte, aspecto especialmente delicado, por lo demás, en relación con cuestiones especialmente sensibles, como son las que atañen, precisamente, al ámbito de la prevención de riesgos laborales. A las dificultades planteadas por este continuo avance tecnológico, se une, por otra parte, la futilidad de buena parte de las soluciones que pudieran articularse ante la intensidad de unas agresiones que resultan, sin duda, explicables, esperables, incluso, hasta cierto punto, en el contexto socioeconómico actualmente vigente, en el que la información se erige, claramente, y de forma cada vez más global, en instrumento de poder, en valor de cambio, desatando un voluminoso y difícilmente controlable tráfico de información.

Ponencia: PREVENCIÓN DE RIESGOS PSICOSOCIALES CAUSADOS POR LAS NUEVAS TECNOLOGÍAS

Ponente: Juan José Fernández Domínguez, Catedrático de Derecho del Trabajo y de la Seguridad Social de la Universidad de León

Las patologías derivadas de la masiva introducción de la tecnología en la vida y en el trabajo mueve a reflexionar sobre las dificultades para la adaptación causadas por la 
falta de habilidad para tratar adecuadamente con tan ingente como imprescindible bagaje.

Bajo el término tecnoestrés se agrupan una serie de desequilibrios funcionales en los que ansiedad, sobrecarga de información, conflicto de roles, factores de organización, ausencia de formación $y$ un largo etcétera se proyectan bajo patrones $o$ denominaciones conocidas, a las cuales viene a añadirse el prefijo o sufijo "tecno" para dotarlos de una nueva dimensión, en el sempiterno dilema derivado de los fallos en la relación "demanda-recursos-control”.

Experiencias pasadas con las TICs, actitudes y valores frente a la tecnología y márgenes y sentimientos de autoeficacia irreal o impotencia ante las innovaciones introducidas en el trabajo se traducen en variados tipos específicos de riesgos, siempre presididos por las constantes de las fuentes tradicionales: demandas técnicas desproporcionadas o desequilibradas en relación con carencias en habilidades personales reales o presuntas (sentidas como tales o sobrevaloradas, admitiendo tanto el exceso como el defecto) y falta de los amortiguadores necesarios (autoeficacia específica, estrategias de afrontamiento o coping y un grado de aceptación normalizado) como factores imprescindibles para hacerles frente.

Ha lugar, así, a la aparición de un escenario donde, entre otros actores, cobran singular protagonismo el síndrome de fatiga informativa (data smog), la locura multitarea (multitasking madness) y la "accidentabilidad informática" (computer hassles); a sus resultas, casi siempre, el síndrome de estar quemado (burnout).

$\mathrm{Su}$ incidencia exige, como primera medida de prevención, contar con instrumentos fiables para detectar el fenómeno general y sus variantes, apoyados en herramientas de evaluación como los cuestionarios CET, RED.es, Red_General, Red_TICC o RED_TT, aun cuando el único validado a día de hoy en España es el RED-TC (Nota Técnica de Prevención 730).

En sus variantes, tres son las manifestaciones básicas con estructura de análisis consolidadas. Habiéndose prestado atención específica a la tecnoadicción (que precisaría ser ponderada en otros contextos o personalidades adictivas), aquí se aludirá a las otras dos con perfil nítidamente delimitado: la tecnoansiedad o tecnofobia y la tecnofatiga.

La tecnofobia puede ser comprendida a través de tres factores básicos: resistencia, miedo o ansiedad y pensamientos hostiles hacia lo tecnológico, de intensidad variable, los cuales llevan desde la incomodidad a la sensación cognitiva, hasta alcanzar el grado patológico de la ansiedad o miedo irracional. En este nivel surgirán los trastornos adaptativos (psicológico, social o de funcionamiento) y la ansiedad generalizada y somatizada a nivel físico y psicológico, para cuyo tratamiento resulta aconsejable recurrir a psicoterapias de exposición: desensibilización sistemática, inundación o terapia implosiva y exposición gradual.

La tecnofatiga, o agotamiento tanto mental como cognitivo, puede ser fruto de la conocida como "infoxicación", o falta de habilidad para procesar toda la información 
de que dispone el usuario. De gravedad manifiesta, en la medida en que su somatización física y mental puede conducir a situaciones incapacitantes o problemas notorios de rendimiento, las medidas de prevención disponibles son las comunes ante cualquier fenómeno de estrés, adaptadas a este contexto específico: organizacionales, o de intervención en las condiciones de trabajo generales, e individuales, que potencien la resistencia allí donde ha fallado la resistencia.

Ponencia: RIESGOS ASOCIADOS A LA UTILIZACIÓN DE PANTALLAS DE VISUALIZACIÓN Y DISPOSITIVOS DE CONTROL

Ponente: José Gustavo Quirós Hidalgo, Profesor Titular de Derecho del Trabajo y de la Seguridad Social de la Universidad de León

Por pantalla de visualización ha de entenderse cualquier pantalla alfanumérica o gráfica capaz de representar texto, números o gráficos, independientemente del método de presentación utilizado. Conviene destacar su frecuente uso en el ámbito laboral, en la actualidad en cualquier sector, pues la tecnología hace que cualquier actividad profesional requiera el uso de equipos informáticos, tablets, smartphones, terminales, etc.

Conforme a la LPRL, el empresario tiene, entre otras, la obligación de adoptar, respecto al personal a su servicio, las medidas necesarias para que la utilización por los trabajadores de los equipos de trabajo --y las pantallas de visualización lo son-- no suponga riesgos para su seguridad o salud o, si ello no fuera posible, para que tales riesgos se reduzcan al mínimo. En este sentido, los principales riesgos asociados a la utilización de estos equipos de trabajo son trastornos musculoesqueléticos, problemas visuales y fatiga física y mental.

Por todo ello, el Real Decreto 488/1997, de 14 de abril, regula las disposiciones mínimas de seguridad y salud relativas al trabajo que incluye pantallas de visualización. Asimismo, existe una Guía Técnica del Instituto Nacional de Seguridad e Higiene en el Trabajo (edición de 2006) para la evaluación de los riesgos inherentes al uso de estos dispositivos y varias Notas Técnicas de Previsión que establecen buenas prácticas relativas a la iluminación, descansos, ergonomía, etc.

En resumen, la evaluación de los riesgos derivados del uso de pantallas de visualización por parte de los trabajadores usuarios y las medidas para evitar o reducir sus riesgos debe atender fundamentalmente a los siguientes elementos: el diseño del puesto de trabajo (medias y dimensiones de sillas, mesas y espacios), los elementos del puesto (pantalla, teclado, superficie de trabajo, asiento), el medio ambiente físico (iluminación, ruido, calor, emisiones, humedad), la relación ordenador-persona (programas o aplicaciones a utilizar) y la organización del trabajo (formación e información, reparto de tareas, pausas). 
Ponencia: EL ACCIDENTE IN ITINERE Y EN MISIÓN EN EL MARCO DE LA INDUSTRIA 4.0

Ponente: Rodrigo Tascón López, Profesor Titular de Derecho del Trabajo y de la Seguridad Social de la Universidad de León

Los increíbles avances tecnológicos que está contemplando la humanidad tienen influencias múltiples sobre los más variados aspectos de la vida social y jurídica. Uno entre muchos es el relativo al desplazamiento de trabajadores, esto es, la necesidad que tienen los operarios de desplazarse, moverse, en el mundo físico, para desarrollar su prestación profesional, ya sea de forma normal o habitual; ya de forma más excepcional, para realizar tareas en lugares distintos al centro de trabajo (viajes en misión).

El Derecho del Trabajo se ha ocupado tradicionalmente de estas cuestiones desde varios puntos de vista: limitaciones a la decisión temporal de desplazamiento de trabajadores y a la definitiva de la movilidad geográfica (art. 40 ET); consideración de accidente de trabajo tanto el sufrido in itinere como el ocurrido en misión (art. 156 LGSS). La industria 4.0 influye, al menos, de dos formas diferentes en los desplazamientos de los trabajadores:

$1^{\circ}$.- La tecnología evita y evitará desplazamientos de los trabajadores: las nuevas herramientas tecnológicas están empequeñeciendo el mundo. Hoy se puede en tiempo real tener una reunión virtual con un cliente o proveedor, enviar gran cantidad de información en cualquier lugar del mundo, perfeccionar contratos a través de firma electrónica, trabajar a distancia (algo que se ha facilitado tras la reforma del art. 13 ET operada por la famosa reforma laboral de 2012, que no todo hizo mal...) haciendo llegar el fruto del esfuerzo a la empresa en el mismo momento en el que es realizado por el teletrabajador que, a su vez, puede ser controlado perfectamente por la empresa a través de dispositivos remotos (que, en principio, y si han sido debidamente informados, son lícitos y no atentan contra derecho fundamental inespecífico alguno)...

Todas estas potencialidades han de llevar a una disminución de los desplazamientos de trabajo porque es un objetivo deseable desde diversos puntos de vista: tanto para el trabajador individual (las limitaciones del art. 40 muestran a las claras que el interés del operario es precisamente no tener que viajar por motivos laborales) para facilitar la conciliación de la vida personal, familiar y laboral; como colectivo (menor impacto ambiental, reducción de atascos...).

$2^{\circ}$.- La tecnología permite nuevas formas de desplazamiento más rápidas y seguras. Y esto puede ser usado por los trabajadores y favorecido y potenciado por las empresas como vía para prevenir posibles siniestros, evitando así responsabilidades civiles privadas y públicas de Seguridad Social (lo que redunda en beneficio de las maltrechas arcas del Sistema).

Por otro lado, el Derecho de la Seguridad Social, encargado de prestar protección a quien sufra un accidente como consecuencia de un desplazamiento profesional, ha experimentado (está experimentando) una constante reconstrucción del concepto de 
accidente de trabajo, consagrado en el art. 156 LGSS, para adaptarlo a las nuevas realidades.

Así, el accidente in itinere, aun cuando sigue requiriendo de sus característicos elementos locativo, teleológico y medial, ha sido flexibilizado en los últimos tiempos por la jurisprudencia para entender, conforme a la realidad social, que se puede compaginar un domicilio familiar con otro profesional, al que viajar la noche antes de la incorporación efectiva al trabajo, quedando el accidente ocurrido en dicho trayecto incorporado al concepto de contingencia profesional.

Por su parte, el accidente en misión ha sido considerado plenamente por la jurisprudencia como accidente de trabajo, aun cuando los pronunciamientos más recientes han explicado que no pueden extenderse sus efectos a los períodos en los que el trabajador estaba disfrutando de períodos de descanso en el hotel o de momentos de ocio en playas o lugares similares, salvo que se pruebe de forma indubitada su relación con el trabajo.

Ponencia: REVOLUCIÓN ROBÓTICA E INTERACTUACIÓN HUMANOS-ROBOTS EN EL TRABAJO EN ESPECIAL, LAS IMPLICACIONES EN TORNO A LA SEGURIDAD Y SALUD EN EL TRABAJO

Ponente: Ma Yolanda Sánchez-Urán Azaña, Catedrática de Derecho del Trabajo y de la Seguridad Social de la Universidad Complutense

\section{CUESTIONES PREVIAS}

- ¿Por qué hablamos de Revolución Robótica?: se advierte que los robots y los sistemas de IA son unos de los grandes inventos disruptivos del entorno digital

\section{PERO}

Contexto: $4^{\mathrm{a}}$ Revolución Industrial: realidad compleja, heterogénea más allá que una simple descripción del cambio impulsado por la tecnología

Distinción entre IA y Robótica (Ciencia?: combinación de muchas disciplinas científicas)

Concepto de IA- "sistemas que muestran un comportamiento inteligente al analizar su entorno y tomar medidas, con cierto grado de autonomía, para alcanzar objetivos específicos" Comunicación de la CE "IA en Europa”, 24-42018

- ¿Por qué Robótica Inclusiva?- Dimensión social, ética, legal...

- . No importancia de las características concretas, intrínsecas de los robotsConcepto técnico de robot

- . Sí importancia de:

Especificidad del contexto, diferentes características de los robots, IA

- Determinar con precisión los retos sustanciales

_ Fijar con precisión los problemas subyacentes profundos 
- EL SER HUMANO EN EL CENTRO: Ética-Derecho: influencias recíprocas Roboética (VERUGGIO, G.)- Conjunto de criterios o teorías para dar respuesta a los problemas éticos derivados del diseño, desarrollo y uso de los robots

Problemas comunes a todas las tecnologías emergentes

- Problemas o cuestiones singulares de la Robótica:

- Relaciones entre seres humanos-máquinas (usos y límites de la robótica)

_ "Status moral de los robots": objeto, agente moral, sujeto

\section{PROYECCIÓN POLÍTICA Y JURÍDICA DE LA ROBÓTICA}

REGULACIÓN - ¿Cuándo, cómo y qué modalidad de regulación?

_ Diferentes enfoques: ¿hard law/soft law?

_ Principio: Innovación tecnológica social y jurídicamente responsable (más allá del principio de precaución y del de neutralidad tecnológica)

Marco Europeo: Pilar Europeo de Derechos Sociales //Equilibrio entre facilitar el desarrollo tecnológico robótico y proteger los valores que son deseados por los humanos

Identidad humana/dignidad y en tono a ellas sus derechos irrenunciables

- Mejora del ser humano a través de la tecnología

_ Respeto a los derechos humanos

¿REGULACIÓN JURÍDICA? - ¿Derecho de la Robótica? ¿Derecho de los Robots?

- Concepto técnico (específico y amplio) de Robot

- Rasgos o caracteres-Interacción física/cognitiva entre humanos-robots

IMPACTOS O PROBLEMAS que requieren respuesta desde del Derecho:

AFECTACIÓN DEL MERCADO DE TRABAJO (Grupo de Alto nivel UE sobre el impacto de la transformación digital en el mercado de trabajo en la UE 2018)

IMPLICACIONES JURÍDICO-LABORALES DE LA ROBOTIZACIÓN Y $\bar{S} O L U C I O N E S$

\section{IMPLICACIONES JURÍDICO-LABORALES DE LA ROBOTIZACIÓN}

\section{A) PUNTO DE PARTIDA}

Confrontación de ideas y proyecciones-tecno-pesimistas -riesgos para el empleo y la ocupación: efecto sustitución de trabajos (destrucción-BOTSOURCING) -tecnooptimistas -oportunidades o impacto positivo neto sobre los trabajos y la calidad del empleo; sustitución de unas tareas concretas y no de otras (en especial las de propiedades humanas)// dudas sobre el incremento de la cantidad y calidad del empleo de los humanos y necesidad de abordar los problemas del período de transición en las condiciones de trabajo y empleo.

Ausencia de una foto fija para todos los sectores, todo tipo de trabajo, todo nivel de habilidades y capacidades, todos los mercados, todos los países...

_ Perspectiva temporal y pronósticos: a largo, medio y corto plazo

_ Brecha digital y su coste social 
_ "Red de seguridad" en el período de transición: Igualdad de oportunidades e integración de colectivos vulnerables (perspectivas de género/edad)

¿Quiénes son los más vulnerables?- Los resultados de las estimaciones muestran que la probabilidad de automatización disminuye con el grado de responsabilidad en la empresa, el nivel educativo, la disposición a participar en acciones formativas y la adopción de nuevas formas de trabajo -como el teletrabajo-, y es comparativamente reducida para los ocupados en educación, sanidad, servicios sociales, TIC, energía y actividades científico-técnicas. Las restantes características del trabajador -como el género, la edad, la nacionalidad, la antigüedad, el tipo de contrato y la situación laboral de procedencia- y de la empresa -como el tamaño- juegan un papel secundario para explicar el riesgo de digitalización-BBVA.

\section{B) MARCO REGULATORIO DE LAS RELACIONES LABORALES}

1) PLANTEAMIENTO: ECUACIÓN ENTRE LIBERTAD EMPRESARIAL Y FUNCIÓN Y FINALIDAD DEL DERECHO DEL TRABAJO: PROTECCIÓN Y GARANTÍA DEL TRABAJO HUMANO

1) Implicación de los empresarios-representantes de los trabajadores

2) Mínima, necesaria, intervención legal imperativa (hard law)

\section{2) PROYECCIONES}

a) Sobre el concepto de trabajador y formas de empleo en el mercado de trabajo robotizado

b) Condiciones de trabajo y el impacto de la robótica. Reflexiones sobre algunos de los problemas. En especial en la relación individual de trabajo

IV. CONDICIONES DE TRABAJO E IMPACTO DE LA ROBÓTICA. EN ESPECIAL SEGURIDAD Y SALUD EN EL TRABAJO

A) Algunas cuestiones previas

- ¿REPLANTEAMIENTO DEL CONCEPTO DE TRABAJADOR PERSONA DISCAPACITADA? ¿Podría alterarse el concepto de discapacidad en un futuro?

- Sobre los robots-workers-Debate sobre la personalidad electrónica o sobre los ECNP-No eliminación del rasgo de "humanidad" en la configuración jurídica del concepto de trabajador -- ¿Se les podrá otorgar un determinado status laboral?-De su consideración como mero instrumento de trabajo, a herramienta de trabajo completa tecnológicamente, ¿también sujeto de derechos $\mathrm{y}$ obligaciones laborales? - AUSENCIA DE VOLUNTARIEDAD

- Cambio del MODELO DE TRABAJO y polarización de los trabajadores (brecha digital y su efecto sobre colectivos específicos de trabajadores)-

- Reparto del trabajo y protección de los trabajadores más vulnerables Desigualdad "digital" y principio de igualdad de oportunidades y no discriminación en el empleo 
B) Condiciones de trabajo y el impacto de la robótica-DOS VERTIENTES O DIMENSIONES: 1) Trabajo colaborativo con robots/2) Efecto sustitución

\section{EFECTO SUSTITUCIÓN}

Derecho a la no discriminación (principal red de seguridad jurídica de los trabajadores Robotización y comportamientos asépticamente ligados a un derecho empresarial a la innovación tecnológica -incidencia en determinados colectivos de trabajadoresCausa de discriminación indirecta.

La causa técnica (derivada de la robotización) debe orientar medidas en relación con la remuneración o con la amortización de puestos de trabajo (o suspensiones o modificaciones).

Botsourcing y sus implicaciones laborales-el robot como mejora técnica y los aspectos de flexibilidad interna y flexibilidad externa (necesidad de especificación en el plano normativo para concretar la razón técnica derivada de la robotización-incorporación masiva de robots-reemplazo de trabajadores y medidas ponderadas y equilibradas limitativas del impacto del despido: necesidad de medidas preventivas a largo plazo y reparadoras a corto plazo
A largo plazo---AJUSTES RAZONABLES (PARA LA READAPTACIÓN DEL PUESTO)-Problema: "carga excesiva para el empresario" (contrarrestado con los incentivos fiscales o subvenciones o bonificaciones)- --Aspecto controvertido "AYUDAS AUXILIARES” (exoesqueletos)

TRABAJO COLABORATIVO- Seguridad y Salud en el trabajo

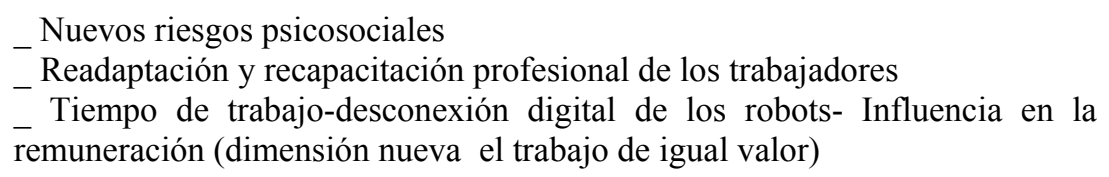

En el campo de la Robótica Industrial se ha ejemplificado cómo los robots colaborativos pueden influir positivamente en la productividad de los trabajadores humanos ${ }^{1}$ porque entre otros efectos pueden incrementar la ergonomía en los procesos y reducir el riesgo de lesiones en el lugar de trabajo, disminuyendo así las bajas

\footnotetext{
${ }^{1}$ Un ejemplo citado en muchas ocasiones es el de la fábrica estadounidense de BMW en Spartanburg donde los robots colaborativos ayudan a colocar puertas con aislamiento de sonido y humedad, una tarea que solía causar tensión en la muñeca de los trabajadores https://roboticsandautomationnews.com/2017/03/04/bmw-shows-off-its-smart-factorytechnologies-at-its-plants-worldwide/11696/

$\mathrm{O}$ el del fabricante de electrónica canadiense Paradigm Electronics, que utiliza robots para llevar a cabo tareas de pulido en los altavoces http://www.manufacturingglobal.com/ technology/collaborative-robotics-increased-paradigm-electronics-throughput-50; los robots han incrementado en un 50\% la productividad pero sin pérdida de empleo de los trabajadores humanos que han sido reconducidos a tareas de acabado final y de control de calidad.
} 
laborales derivadas de acciones repetitivas físicas o de procesamiento de datos y reduciendo la accidentalidad laboral.

Pero también preocupa desde esta perspectiva, la de seguridad y salud en el trabajo, y pensando en términos de colaboración, interactuación o trato con robots en el trabajo (no la perspectiva de reparto separado de tareas), la diferencia que puede haber entre tener a humanos como compañeros de trabajo a tener a robots, sobre todo cuando éstos tengan incorporados cada vez mayores dosis de IA, lo que indudablemente generará nuevos riesgos psicosociales para el trabajador humano derivados de la impredecibilidad de actuación de estas máquinas sofisticadas.

\section{MINORACIÓN DE LA EXPOSICIÓN AL RIESGO---ROBÓTICA INDUSTRIAL - Los robots como medios para cumplir las normas sobre seguridad y salud en el trabajo y disminución de riesgos \\ NUEVAS PERSPECTIVAS:}

a. Robots inteligentes

b. Cooperación/interactuación humanos-robot

Lo que quiere decir que en materia de prevención de riesgos laborales, así como las normas establecen reglas o medidas de control y prevención atendiendo a colectivos específicos de personas (edad, discapacidad, maternidad), habrá que establecer también medidas preventivas orientadas a definir el riesgo y los derechos y obligaciones cuando aparezca un robot de mayor autonomía. No solo por tanto medidas de seguridad, tal y como se establecen en la industria, sino orientando la prevención hacia nuevos riesgos psicosociales para los trabajadores humanos que interactúan con robots de esas características.

Condiciones o requisitos de seguridad---Si los robots son equipos de trabajo--¿cómo se debe adaptar la normativa en particular el RD 1215/1997 a estos equipos?

Si los robots cooperadores/inteligentes son más que un medio o equipo de trabajo--¿podrían incrementar los riesgos psicosociales?---¿cómo afrontar la protección y garantía de la salud psicológica y social de los trabajadores?

¿Serían los robots un elemento o factor de riesgo en sí mismo?-Un nuevo factor de riesgo como la organización del tiempo de trabajo, por ejemplo.

El robot puede reducir riegos físicos de muchas actividades... pero puede producir otros - mayor presión psíquica, por ejemplo, con aumento del estrés laboral y la fatiga

Adecuación de quién a quién en los ritmos de trabajo, en los tiempos de descanso, mayor responsabilidad de trabajadores, aislamiento del trabajador que interactúa más con robots y menos con humanos, ...: tecnoestrés, tecnofobia, tecnofatiga...

Enlaza esta perspectiva con la de readaptación y recapacitación de los trabajadores y con la visión de la necesaria búsqueda de equilibrio entre derechos de los trabajadores y las cargas para los empresarios. Si la formación y recapacitación profesional es un derecho del trabajador, cada vez más amplio en nuestra legislación, una nueva perspectiva se abre cuando se trata de medir el impacto de la robótica en esta 
dimensión de derechos laborales porque no puede olvidarse que, para que el trabajador pueda beneficiarse de las oportunidades que ofrece la robótica (y en particular la equiparación de la generación actual de trabajadores a las futuras), se requiere que haya una inversión efectiva en formación y capacitación, no solo de los poderes públicos, sino también de las propias empresas, que exigirán que los ajustes razonables no supongan una "carga excesiva".

¿Es suficiente la formación técnica del trabajador?

¿Debe incorporarse en los convenios colectivos?: ver CC Repsol, CC Bridgestone, CC industria química

Medidas necesarias: nivel formación de los trabajadores, formación continua, regulación de la jornada de trabajo, normas que regulen el uso de robots en nuevas áreas de actividades

Otra de las dimensiones del derecho a la seguridad y salud en el trabajo es la que concierne al tiempo de trabajo, en particular desde una perspectiva comunitaria, puesto que la Directiva UE sobre tiempo de trabajo (Directiva 2003/88) se ha ideado como instrumento legal de garantía de aquel derecho. Por tanto, la robotización de trabajos y tareas y la interactuación humano-robot exige plantear si más allá de la reducción de jornada de trabajo como alternativa al posible desempleo tecnológico ${ }^{2} \mathrm{o}$ la propuesta o programas piloto ideados en algunos países (tal es el caso de Suecia, que propuso una jornada de 6 horas al $\mathrm{dia}^{3}$ ) para a ayudar a trabajar a más personas, haya que pensar también en la "desconexión digital" de los robots, es decir, en la medición de "su" tiempo de trabajo en relación o proporción a la media de los humanos (no a la inversa) para repartir con éstos la que es o se percibe como jornada habitual; y ello aunque se avance hacia una concepción flexible de la distribución irregular del tiempo de trabajo. Dicho de otra forma, y preguntándonos, ¿Habría que reconfigurar el tiempo de trabajo teniendo en cuenta el máximo que habría que asignar a los robots en las empresas? Se podría hablar de una desconexión robótica en las empresas como derecho de los trabajadores para un reparto equitativo del tiempo de trabajo entre robots y trabajadores humanos; aspecto éste, que influirá en la remuneración. Sin perjuicio de nuevas y multiples formas de retribución que al respecto puedan establecerse, hay que plantear un dimension nueva del principio de igual retribución por trabajos de igual valor, que exigirá establecer reglas para medir o intentar objetivar los criterios para determinar que el trabajo realizado por los humanos es de igual valor que el trabajo realizado por los robots no tanto con fundamento en elementos cuantitativos (porque ello repercutiría negativamente en los humanos) como cualitativos que corresponderá describir en acuerdos y convenios

2 Recuérdese que Keynes ya predijo que el reemplazo de trabajadores humanos por máquinas obligaría a una reducción progresiva de la semana laboral, que el fijaba en una proyección hacia 2030 de 15 horas; KEYNES, M. (1933) Economic possibilities for our grandchildren. En: Essays in persuasion. p. 358-73).

3 GREENFIELD, R.: "How the Six-Hour Workday Actually Saves Money", Bloomberg, April 17, 2017, available at: https://www.bloomberg.com/news/articles/2017-04-17/how-the-sixhour-workday-actually-saves-money. After a 2 years trial found that people working the 6 hours shift took 4.7 percent fewer sick days and had a higher level of well-being, however, due to lack of funding the study was stopped. 
colectivos con apreciación detallada de las capacidades humanas, sea en trabajos industriales o de servicios.

Por último, control, supervisión y responsabilidad:

.Robots con capacidades de control diagnóstico y solución de problemas: repercusión en mandos intermedios y de supervisión

.Robots y control de riesgos: el robot como medida preventiva-Ejemplo exoesqueletos (aumento de capacidades; mejora cuantitativa y cualitativa del trabajo) -relación con el concepto de discapacidad/ajustes razonables/ efecto en el derecho a la no discriminación y reducción de la brecha e integración de personal femenino en tareas que exigen un esfuerzo físico paliado por la robótica: teoría del "trabajador aumentado"

.Responsabilidad: de nuevo la concepción del robot, ¿es un mero instrumento o apoyo técnico en el trabajo?-Influencia en la responsabilidad del empresario y, en particular, en los accidentes de trabajo provocados por robots

STS de 20 de enero de 2010-responsabilidad por AT provocado por robots Otros países: Francia/Alemania: reglas generales sobre prevención de riesgos laborales

\section{Ponencia: TRABAJADORES CON DISCAPACIDAD Y NUEVOS RIESGOS EN LA} INDUSTRIA 4.0

Ponente: Henar Álvarez Cuesta, Profesora Titular de Derecho del Trabajo y de la Seguridad Social de la Universidad de León

El sistema productivo está transformándose (lleva haciéndolo mucho tiempo) merced a la cuarta revolución industrial. Esta industria 4.0 o "industria inteligente" supone un salto cualitativo en la organización y gestión de la cadena de valor del sector; e implica la incorporación de las nuevas tecnologías (Big data y análisis de datos, Cloud Computing -evolucionando desde los datos en la nube a los datos en la niebla-, ciberseguridad, robótica, internet de las cosas, simulación y prototipado, realidad aumentada, etc.) a la considerada "tradicional", permitiendo que dispositivos y sistemas colaboren entre ellos y con otros para crear una industria inteligente. La industria, al tiempo, transforma la economía tradicional en digital, y adquiere cuatro características específicas: la irrelevancia de la ubicación geográfica, el papel clave de las plataformas, la importancia de los efectos de red y el uso de grandes datos.

Los anclajes de la cuarta revolución industrial redimensionan, en su conjunto, los procesos de automatización y digitalización; vuelven a profundizar en las consecuencias del cambio tecnológico, y complejizan aún más las dimensiones del mismo hasta llegar a la esfera laboral.

El menor de los cambios no ha sido (ni será) la incorporación de robots con inteligencia artificial. Los retos a los que se enfrenta la prevención de riesgos pasarán en el futuro por la introducción de tecnologías de mejora humana como los exoesqueletos o robots portátiles, visión artificial o neurochips, que generarán nuevos o remozados riesgos a investigar, evaluar y prevenir; los arreglos ergonómicos y 
logísticos de los robots autónomos necesitan nuevas pruebas y actividades de pilotaje en las industrias y en el sector de servicios y se deben ofrecer programas de formación adaptados a los trabajadores que programarán, explotarán, mantendrán o compartirán el lugar de trabajo con estos robots; en fin, los efectos de la robótica sobre la motivación y el bienestar de los trabajadores y directivos no son ampliamente conocidos y por ello los factores psicosociales relacionados con la robótica requerirán más atención en el campo de la seguridad y la salud.

En el futuro, la cooperación entre robots y humanos se diversificará, con los robots aumentando su autonomía y la colaboración entre humanos y robots alcanzando formas completamente nuevas. Los enfoques actuales y las normas técnicas destinadas a proteger a los empleados del riesgo de trabajar con robots colaborativos tendrán que ser revisados, ampliados y desde luego incorporados a los planes de prevención.

Desde la Unión Europea se reclama la instauración de sistemas globales que garanticen la protección de la salud y la seguridad de los trabajadores que operan con o junto a sistemas robotizados u otras formas de inteligencia artificial, así como la introducción de normas que regulen las responsabilidades y garanticen que los daños causados por robots autónomos puedan dirimirse en favor de los trabajadores;

Si el uso de robots en la producción plantea importantes desafíos para la salud y la seguridad en el trabajo, los retos se intensifican y precisan de estudio propio para los empleados con discapacidad. Por un lado, la robotización puede reducir la carga física sobre los trabajadores, pero también puede dar lugar, por otra parte, a mayores tensiones psíquicas y a la necesidad de una protección específica como consecuencia de la creciente responsabilidad humana en procesos de producción más complejos.

En el primer sentido, los mayores beneficios derivados del uso más amplio de la robótica deberían ser la sustitución de personas que trabajan en entornos poco saludables o peligrosos y la utilización de estos robots para eliminar o paliar la discapacidad padecida. En el espacio, la defensa, la seguridad o la industria nuclear, pero también en logística, mantenimiento e inspección, los robots autónomos son particularmente útiles para reemplazar a los trabajadores humanos que realizan tareas sucias, aburridas o inseguras, evitando así la exposición de los trabajadores a agentes $\mathrm{y}$ condiciones peligrosas $\mathrm{y}$ reduciendo los riesgos físicos, ergonómicos $\mathrm{y}$ psicosociales.

En el segundo, y de acuerdo con lo previsto en el art. 25 LPRL, el empresario ha de tener en cuenta la discapacidad de sus trabajadores en las evaluaciones de los riesgos $\mathrm{y}$, en función de éstas, adoptar las medidas preventivas y de protección necesarias. Asimismo, estos trabajadores no deben ser empleados en aquellos puestos de trabajo en los que, a causa de sus características personales, estado biológico o por su discapacidad física, psíquica o sensorial debidamente reconocida, puedan ellos, los demás trabajadores u otras personas relacionadas con la empresa ponerse en situación de peligro o, en general, cuando se encuentren manifiestamente en estados o situaciones transitorias que no respondan a las exigencias psicofísicas de los respectivos puestos de trabajo. La introducción de robots con inteligencia artificial o 
la utilización de dispositivos tecnológicos modificará y dotará de nuevo contenido a este mandato legal.

Ponencia: RIESGOS INHERENTES A LA DIGITALIZACIÓN DEL TRABAJO: NUEVOS HORIZONTES PARA LA PREVENCIÓN DE RIESGOS LABORALES

Ponente: Javier Fernández-Costales Muñiz, Catedrático EU (integrado TU) de Derecho del Trabajo y de la Seguridad Social de la Universidad de León

La disciplina preventiva forma parte del contrato de trabajo desde el punto de vista de los riesgos que genera el hecho de desarrollar una prestación de servicios, peligros que requieren de una protección específica, centrada y orientada básicamente a eliminar, evitar o reducir en el mayor grado de los posible no sólo esos riesgos, sino también las consecuencias que su materialización pueda llegar a tener, objetivo presente en las legislaciones de la mayor parte de los Estados, que establecen, regulan e imponen obligaciones, límites y responsabilidades atendiendo a las acciones (o inacción) de las partes en virtud de unos determinados estándares de seguridad que varían ostensiblemente de unos territorios a otros.

El lugar de trabajo puede cambiar a lo largo de la vigencia del contrato, entre otras razones porque el trabajador tiene una cierta movilidad espacial, pudiendo su prestación exigir hacer uso de la misma en mayor o menor intensidad. El desplazamiento transnacional de trabajadores en el desarrollo de una prestación de servicios, acontecimiento que en los últimos tiempos ha visto evolucionar y crecer exponencialmente su incidencia en un mundo cada vez más globalizado, donde el enorme avance de las nuevas tecnologías y los medios de comunicación han permitido reducir sustancialmente las distancias que antaño suponían un claro obstáculo en este ámbito.

El artículo 3.1.e) de la Directiva 96/71, reformada por la nueva Directiva (UE) 2018/957 del Parlamento Europeo y del Consejo, de 28 de junio de 2018, aplicable a los trabajadores desplazados en una prestación de servicios transnacional obliga a los Estados miembros a velar, cualquiera que sea la legislación aplicable a la relación, por que las empresas que realizan el desplazamiento garanticen, entre otras, las condiciones de trabajo y empleo y de seguridad e higiene en el trabajo, destacando, por su parte, el artículo $4.7^{\circ}$ LPRL que se entenderá por condición de trabajo "cualquier característica del mismo que pueda tener una influencia significativa en la generación de riegos para la seguridad y salud del trabajador", lo que abarca, indudablemente, el desplazamiento transnacional para prestar servicios en otro país.

Las disposiciones en materia de seguridad y salud en el trabajo del país de destino parece, en principio, que deberán situarse entre las normas a respetar por la empresa que desplaza a su empleado, en tanto en cuanto en esta materia se procedió, como se ha visto, a realizar una armonización de las diferentes legislaciones de los estados miembros, de forma tal que existe una protección estándar mínima en el ámbito preventivo que acerca las distintas normativas y las hacen en buena medida muy similares en sus bases, aun cuando cabe señalar que evidentemente no todos los países 
adaptaron de manera idéntica ni con el mismo alcance regulador la directiva marco, o no vigilan con la misma intensidad su aplicación, produciéndose diferencias que provocan la antes mencionada dificultad en muchos momentos a la hora de determinar el cuerpo regulador de aplicación.

Ponencia: PREVENCIÓN DE RIESGOS LABORALES EN LA INDUSTRIA 4.0 ¿ES POSIBLE UN ANÁLISIS DE GÉNERO?

Ponente: Beatriz Agra Viforcos, Profesora Titular de Derecho del Trabajo y de la Seguridad Social de la Universidad de León

1. Industria y Trabajo 4.0

- Desarrollo del software, IA, robots, conexión persona-máquina... i $i^{\mathrm{a}}$ Revolución Industrial?

- $\quad$ Pilares de la Industria 4.0:

○ El tecnológico (internet de las cosas, realidad virtual, simulación productiva...)

- El humano (formación cualificada y multidisciplinar, cultura del trabajo...)

○ El organizativo (terciarización, colaboración, globalización, fragmentación...)

- La Industria 4.0 genera un Trabajo 4.0, definido por cuatro ejes:

○ Trabajo centrado en lo digital y la conectividad. El centro de trabajo se desdibuja

- Trabajo fragmentado y descentralizado. Se incrementa la individualización de las relaciones laborales o se produce, directamente, una deslaboralización.

- Trabajo flexible y con horarios no claramente delimitados

- Trabajo para cuyo desarrollo son precisos nuevos perfiles profesionales

2. Impacto de la robótica y la digitalización en el trabajo

- Generan procesos de destrucción y creación de empleo y efectos en las condiciones de trabajo

- $\quad$ Surgen nuevas profesiones y actividades y desaparecen otras.

- Importancia de la educación, el reciclaje profesional y el aprendizaje a lo largo de la vida

- Segmentación del mercado de trabajo y precarización de los afectados por la brecha digital

- Crisis del Derecho Laboral al desdibujarse el tipo de trabajo sobre el que se edificó

- $\quad$ Efectos en la seguridad y salud en el trabajo

3. Seguridad y salud en el trabajo en la era tecnológica

- Conexión histórica y actual entre tecnología y seguridad y salud en el trabajo

- Nuevas tecnologías y protección del trabajador frente al riesgo laboral:

- Sustitución del hombre por el robot en trabajos peligrosos

- Nuevos medios de prevención y protección individual y colectiva

o Potencial en lo relativo a la formación 
- Potencial en el ámbito sanitario

○ Potencial de redes sociales, blogs, aplicaciones...

○ Mejoras en la gestión (evaluación en tiempo real, inmediatez...)

- Riesgos laborales derivados de las nuevas tecnologías en la Industria 4.0

- Riesgos derivados de la automatización y la robotización

- Riesgos asociados a PVD

- Riesgos asociados al teletrabajo

- Riesgos psicosociales del Trabajo 4.0. Tecnoestrés

4. La Industria y el Trabajo 4.0 en la normativa preventiva

- Referencias en la LPRL y el RSP a la importancia de las tecnología y los avances técnicos

- Reales Decretos vinculados a la cuestión

- Referencias a algunas formas de Trabajo 4.0: temporal, concurrencia de empresarios, contratas, ¿trabajo autónomo?

5. ¿Es posible un análisis de género?

- Valoración de diferencias biológicas hombre-mujer frente a los riesgos laborales:

- Embarazo y lactancia

- Otras diferencias biológicas

- Valoración de diferencias de género frente a los riesgos laborales:

- Doble jornada de trabajo

- Precariedad laboral

- Segregación ocupacional horizontal

- Segregación ocupacional vertical

○ Discriminación como riesgo psicosocial

- Conclusiones

Ponencia: LA PREVENCIÓN DE LOS RIESGOS LABORALES DE LOS TRABAJADORES AUTÓNOMOS Y LAS NUEVAS TECNOLOGÍAS

Ponente: $\mathbf{M}^{\mathrm{a}}$ de los Reyes Martínez Barroso, Catedrática de Derecho del Trabajo y de la Seguridad Social de la Universidad de León

El perfil del trabajo autónomo presenta una significativa diversidad, debido entre otros factores a la emergencia del trabajo autónomo económicamente dependiente, como figura laboral que constituye todavía un porcentaje menor del conjunto del trabajo autónomo, pero cuyas características suponen un cambio cualitativo respecto de la concepción tradicional del mismo. La aparición de esta figura hunde sus raíces en los cambios estructurales registrados en el mundo del trabajo en las últimas décadas, impulsados por una serie de factores, entre los que destaca el crecimiento de los servicios y las oportunidades nuevas que brindan las tecnologías de la información y de la comunicación. A todo ello se suma de forma más reciente el impacto de la digitalización, en una doble dimensión: De un lado, porque las nuevas formas de empleo digital (como el crowd work y el trabajo a demanda vía apps) están creciendo rápidamente en sectores económicos que ya registraban con anterioridad una presencia significativa del trabajo autónomo dependiente, tales como las industrias creativas, transporte, consultorías y los servicios de limpieza. De otro, y de forma más relevante, porque las nuevas plataformas 
on line para el trabajo digital están contribuyendo a potenciar la ambigüedad de la relación laboral por una doble vía: mediante la incorporación de terceras partes en lo que previamente había sido un acuerdo bilateral, planteando la cuestión de quién debería ser considerado como empleador (el cliente, la plataforma digital, otro intermediario...) y creando nuevas asimetrías en el mercado de trabajo, que hacen prácticamente imposible aplicar el concepto de "negociación significativa" implícito en los planteamientos tradicionales de relación laboral entre empleado y empleador, o de contrato de servicio entre trabajador autónomo y cliente.

Y todo ello sin mencionar la existencia del "falso autónomo", fenómeno que por su propia naturaleza irregular no es fácil de cuantificar y que podría haber registrado un cierto crecimiento en los últimos años en el contexto de la crisis económica. En definitiva, cada vez más profesionales autónomos trabajan por cuenta de empresas que ya no contratan a trabajadores. El trabajador asalariado ya no es la forma "óptima" de trabajo para muchas empresas, ni para sus colaboradores; de ahí la necesidad de que las formas innovadoras de trabajo asalariado puedan adaptarse relativamente a las actividades lucrativas que desarrollan ciertas plataformas digitales. Es más, en esta rápida enumeración, quizás habría que mencionar también al "emprendedor digital" o "emprendedor Online", conocida como aquella persona que emprende negocios y proyectos con fines de lucro y de diversa índole a través de Internet. Los emprendedores digitales son el fruto de la globalización y el constante avance de las tecnologías de la información y las redes.

Sin negar la necesidad de continuar enfrentándose a los peligros tradicionalmente tomados en consideración, es preciso incorporar también en este ámbito, entre el elenco de riesgos a erradicar o minorar, los denominados psicosociales, relacionales y/u organizacionales, capaces de afectar también a la salud mental de los profesionales autónomos. A ello contribuye, sin duda, la innovación tecnológica, que además de contribuir a mejorar la producción puede incidir de forma negativa sobre la estabilidad psicológica de los profesionales como consecuencia de la sobrecarga mental y del miedo a lo desconocido. Si bien la presencia de tales riesgos es debida a múltiples factores (desde los medios concretos utilizados en el proceso productivo hasta factores relacionales de difícil control), la ponencia tratará de profundizar en el estudio de las circunstancias de la actividad por cuenta propia en la gestación del riesgo concreto, pues sin lugar a dudas constituye un factor decisivo en la presencia y materialización del riesgo psicosocial.

MESA REDONDA: DIGITALIZACIÓN Y PREVENCIÓN DE RIESGOS LABORALES EN CASTILLA Y LEÓN

TELETRABAJO EN EL MARCO DE LA INDUSTRIA 4.0: ALGUNAS CUESTIONES EN ORDEN A LA PREVENCIÓN DE RIESGOS LABORALES

Natalia Ordoñez Pascua, Ayudante Doctor de Derecho del Trabajo y de la Seguridad Social de la Universidad de León

La conocida como $4^{\mathrm{a}}$ Revolución Industrial o Industria 4.0, con la fuerte irrupción en el mundo laboral y empresarial de las nuevas tecnologías -robótica, inteligencia 
artificial, nanotecnología, analítica, entre otras- ha supuesto una radical modificación en la forma de desarrollo de las relaciones industriales, en las cuales se incardinan nuevos modelos de llevar a cabo la prestación laboral ajustados a las necesidades empresariales concretas.

Por cuanto aquí importa, una de las principales consecuencias del desarrollo tecnológico ha sido el incremento del llamado teletrabajo o trabajo a distancia, que surge como una opción válida y a priori ventajosa para el trabajador y el empresario que cabría resumir -a grandes rasgos- y en orden al primero, en una mayor adaptación de su jornada laboral a su situación familiar, lo cual supone grandes mejoras en la conciliación de vida familiar y laboral y en la calidad de vida, repercutiendo en una mayor satisfacción y productividad. Por cuanto hace al empleador, los beneficios más evidentes aparecen como una reducción de los costes fijos y aumento de la productividad. A todo ello cabe añadir la mejora medioambiental que supone la existencia de menores desplazamientos y su repercusión a nivel contaminante.

El desarrollo de la actividad laboral fuera de las instalaciones del empresario no supone que éste quede eximido de velar por la salud de los trabajadores que por cuenta ajena tiene a su servicio de igual forma que lo hace respecto de aquellos que trabajan físicamente en las mismas. En este sentido, el art. 13 de LET se encarga de recoger los extremos fundamentales en torno al desarrollo del trabajo a distancia, entre los cuales y de manera ineludible hace referencia al derecho de los trabajadores que ejercen su actividad laboral bajo esta modalidad a una adecuada protección en materia de seguridad y salud resultando de aplicación, en todo caso, lo establecido en la Ley 31/1995, de 8 de noviembre, de Prevención de Riesgos Laborales, y su normativa de desarrollo.

Los beneficios y ventajas que abanderan al teletrabajo como su "flexibilidad" y "libertad" pueden tornarse en su mayor debilidad, dejando abierto un extenso abanico de riesgos psicosociales, además de los físicos asociados al empleo de las tecnologías de información y comunicación (uso continuo de pantallas de visualización, telefonía...). En este sentido uno de los principales problemas es la gestión del tiempo de trabajo ya que en los límites entre la vida laboral y profesional se difuminan al estar en uso permanente de los mismos dispositivos en las actividades de trabajo y ocio (ordenadores, tabletas, teléfonos móviles...).

Bajo el panorama descrito y en orden poner en marcha adecuados mecanismos de prevención cabría valorar dos situaciones distintas: por un lado, aquellas en las cuales la prestación laboral se desarrolla dentro del ámbito del domicilio particular del trabajador donde la intromisión y vigilancia empresarial presenta mayores dificultades; por otro, aquellas en las que la actividad es llevada a cabo en determinados centros que si bien se encuentran fuera de las instalaciones empresariales en sentido estricto tienen una vinculación con la empresa que las hacen susceptibles de un mayor control. 
Ponencia: LA PREVENCIÓN DE RIESGOS DE QUIENES TRABAJAN A TRAVÉS DE PLATAFORMAS DIGITALES EN CASTILLA Y LEÓN

Ponente: Francisco Xabiere Gómez García, Personal Investigador en Formación

(JCyL-FSE) de la Universidad de León

El auge de los procesos de digitalización está revolucionando nuestra sociedad y, por ende, las relaciones laborales. Una de las novedades de la denominada «industria 4.0» reside en la expansión de la «economía de plataformas», hasta ahora mal llamada «economía colaborativa». Estas plataformas, las cuales operan vía web o App, permiten a los ciudadanos ofrecer servicios, a la vez que fomentan nuevas oportunidades de empleo, fuentes de ingresos inéditas y originales modalidades de trabajo flexibles.

Pero, de la misma forma, manifiestan un lado oscuro, creando problemas para la aplicación de los marcos jurídicos existentes al desafiar los límites establecidos entre consumidor y proveedor, trabajador por cuenta propia y por cuenta ajena o la prestación profesional y no profesional de servicios. La nítida definición de estas situaciones es precisamente la clave que va a permitir determinar las responsabilidades en materia de prevención de riesgos laborales, impidiendo así el peligro de que se puedan aprovechar las zonas grises reglamentarias para eludir unas normas que fueron diseñadas para proteger, no solo la salud de todos los trabajadores, sino también el interés público.

Estas plataformas digitales se pueden dividir en dos tipos, en función de que las tareas a realizar requieran, o no, la presencia física en el lugar de la prestación de servicios. Así, en Castilla y León son más accesibles las segundas, englobadas dentro del anglicismo crowdwork, por cuanto la llamada a la realización del servicio demandado se realiza en forma de convocatoria global hacia una multitud de posibles trabajadores, donde cada persona que cumpla los requisitos mínimos podría afrontar la tarea, siendo las más habituales las relacionadas con el diseño, la programación, o la traducción. En cambio, las que realizan el trabajo «bajo demanda» con una ejecución local (las más comunes son las dedicadas a la movilidad, el transporte de mercancías y el reparto de comida a domicilio), dependen de la expansión geográfica decidida por la propia plataforma, por lo cual su actual impacto en nuestro territorio autonómico es mucho menor, a excepción de la plataforma de reparto de comida a domicilio Just Eat.

Por cuanto a los riesgos laborales se refiere, hay riesgos comunes para ambas modalidades, como son los derivados de un trabajo informal, precario, incierto y generalmente retribuido a destajo; los asociados al término «tecno-estrés» (que pueden producir tecno-ansiedad, nomofobia, etc.); o la presión por ver su desempeño evaluado públicamente a nivel mundial. Pero también existen riesgos específicos para cada una de las modalidades, siendo similares los del crowdwork a algunos de los del teletrabajo (uso de pantallas, ergonomía, aislamiento, etc.), mientras que los del ondemand work dependen más del sector de actividad concreto, aunque casi todos incluyen la geolocalización continua o el mayor riesgo de accidentes. 
Todavía es demasiado pronto para evaluar el impacto real en la salud de los trabajadores que realizan su prestación mediante las plataformas digitales, pero tanto el creciente número de plataformas existentes como de personas que acuden a ellas en busca de la realización de algún tipo de trabajo remunerado, aconsejan poner más atención en las condiciones laborales en este ámbito, especialmente en aquellas ligadas a la protección de la salud y seguridad en el trabajo.

Ponencia: LOS PROCESOS SOBRE RESPONSABILIDAD POR INCUMPLIMIENTOS EN MATERIA DE PREVENCIÓN DE RIESGOS LABORALES Ponente: Ignacio González del Rey Rodríguez, Catedrático de Derecho del Trabajo y de la Seguridad Social de la Universidad de Oviedo

\section{Alterum non laedere}

1. Los riesgos del trabajo y la protección de la persona trabajadora como elementos esenciales y originarios del ordenamiento social

- Producción industrial y trabajo asalariado: nuevos riesgos y finalidad protectora del Derecho del Trabajo. Desde las primeras "leyes de fábricas": trabajo infantil (1873), tiempo de trabajo (1919) y accidentes de trabajo (1900). Línea de tendencia y evolución constante del ordenamiento laboral (paradigmáticamente PRL e igualdad y no discriminación). El suelo del Derecho (también del Derecho del Trabajo): los derechos fundamentales, de ciudadanía o de la personalidad. "Flexisegurdad": ciudadanos vs trabajadores.

2. Un siglo en la prevención y protección frente a los riegos laborales: de la Ley de accidentes de trabajo de 1900 a la Ley de prevención de riesgos laborales de 1995

- Hacia una responsabilidad objetiva (por riesgo) y tasada (contingencias y prestaciones determinadas). Hacia una responsabilidad socializada (aseguramiento obligatorio) y publificada (Seguridad Social). Instrumentos correctores o complementarios de la protección social de los riesgos profesionales: mejoras voluntarias, recargos de prestaciones, y responsabilidad civil e indemnizaciones por daños. El redescubrimiento de la responsabilidad por daños.

3. Responsabilidades en materia de prevención de riesgos laborales y por contingencias profesionales en el ordenamiento español vigente

- Pluralidad de tutelas: preventiva, resarcitoria (prestacional e indemnizatoria y posibles mejoras), sancionadora (administrativa, penal y disciplinaria) y mixta (recargos). Pluralidad de marcos jurídicos: LPRL, LISOS, LGSS, LETT, LETA, CC, CP... Pluralidad de sujetos obligados y responsables: empresario principal y empresas colaboradoras; promotores y propietarios de obras; servicios de prevención, auditoría y formación; administradores, directivos o encargados; técnicos y responsables de prevención; fabricantes, suministradores e importadores; mutuas y aseguradoras, etc. Pluralidad de responsabilidades empresariales por incumplimientos en materia preventiva: por riesgo o por resultado, punitivas o resarcitorias (y mixtas), directas o derivadas (y 
compartidas). Sanciones administrativas (LISOS), sanciones penales (arts.316 a 318 CP), prestaciones de Seguridad Social (art.242 y 244 LGSS), recargos de prestaciones (art.164 LGSS) e indemnizaciones por daños (arts.1.101 y 1.902, y ss. CC). Pluralidad de procedimientos, de procesos y ¿de jurisdicciones?

4. Prevención y responsabilidad por riesgos profesionales en la Ley reguladora de la jurisdicción social de 2011

- Cambios significativos respecto de sus precedentes: del silencio de la LPL de 1995 a las numerosas previsiones de la LJS de 2011. Competencia omnicomprensiva de la jurisdicción social en materia de prevención y responsabilidades por riesgos laborales desde el punto de vista objetivo (art.2.b), e) y q) LJS): prevención, protección y sanción. Y acumulación de acciones, procesos y recursos (arts.25, 28.2, 30 y 234 LJS). Competencia omnicomprensiva de la jurisdicción social en materia de prevención y responsabilidades por riesgos laborales desde el punto de vista subjetivo (art.2.b), e) y q) LJS): trabajadores y funcionarios, empresas y terceros. Y actos preparatorios y legitimación ampliada. Medidas cautelares (arts.79.5 y 142 LJS): inespecíficas. Distribución de la carga de la prueba (art.96.2 LJS): ¿responsabilidad cuasi objetiva? Proceso de impugnación de actos administrativos en materia de prevención (arts.151 y 152 LJS): sanciones y paralización de trabajos por riesgo grave e inminente para la seguridad y la salud.

5. Modalidades procesales y otras dudas pendientes

- Prestaciones y recargos: proceso de Seguridad Social. Impugnación de sanciones: proceso de impugnación de actos administrativos. Impugnación de recargos: proceso de Seguridad Social. Resarcimiento: ¿proceso ordinario o tutela de derechos fundamentales? ¿Procesos preventivos?: ¿proceso ordinario, conflicto colectivo, tutela de derechos fundamentales o Seguridad Social? Indemnizaciones (DF 5 ${ }^{\mathrm{a}}$ LJS). Cosa(s) Juzgada(s). 